\title{
Predicting and analyzing DNA-binding domains using a systematic approach to identifying a set of informative physicochemical and biochemical properties
}

Hui-Lin Huang ${ }^{1,2}$, I-Che Lin ${ }^{1}$, Yi-Fan Liou ${ }^{2}$, Chia-Ta Tsai ${ }^{2}$, Kai-Ti Hsu², Wen-Lin Huang ${ }^{3}$, Shinn-Jang Ho ${ }^{4}$, Shinn-Ying $\mathrm{HO}^{1,2^{*}}$

From The Ninth Asia Pacific Bioinformatics Conference (APBC 2011)

Inchon, Korea. 11-14 January 2011

\begin{abstract}
Background: Existing methods of predicting DNA-binding proteins used valuable features of physicochemical properties to design support vector machine (SVM) based classifiers. Generally, selection of physicochemical properties and determination of their corresponding feature vectors rely mainly on known properties of binding mechanism and experience of designers. However, there exists a troublesome problem for designers that some different physicochemical properties have similar vectors of representing 20 amino acids and some closely related physicochemical properties have dissimilar vectors.

Results: This study proposes a systematic approach (named Auto-IDPCPs) to automatically identify a set of physicochemical and biochemical properties in the AAindex database to design SVM-based classifiers for predicting and analyzing DNA-binding domains/proteins. Auto-IDPCPs consists of 1) clustering 531 amino acid indices in AAindex into 20 clusters using a fuzzy c-means algorithm, 2) utilizing an efficient genetic algorithm based optimization method IBCGA to select an informative feature set of size $m$ to represent sequences, and 3) analyzing the selected features to identify related physicochemical properties which may affect the binding mechanism of DNA-binding domains/proteins. The proposed Auto-IDPCPs identified $m=22$ features of properties belonging to five clusters for predicting DNA-binding domains with a five-fold cross-validation accuracy of $87.12 \%$, which is promising compared with the accuracy of $86.62 \%$ of the existing method PSSM-400. For predicting DNA-binding sequences, the accuracy of $75.50 \%$ was obtained using $m=28$ features, where PSSM-400 has an accuracy of $74.22 \%$. Auto-IDPCPs and PSSM-400 have accuracies of $80.73 \%$ and $82.81 \%$, respectively, applied to an independent test data set of DNA-binding domains. Some typical physicochemical properties discovered are hydrophobicity, secondary structure, charge, solvent accessibility, polarity, flexibility, normalized Van Der Waals volume, pK (pK-C, pK-N, pK-COOH and pK-a(RCOOH)), etc.

Conclusions: The proposed approach Auto-IDPCPs would help designers to investigate informative physicochemical and biochemical properties by considering both prediction accuracy and analysis of binding mechanism simultaneously. The approach Auto-IDPCPs can be also applicable to predict and analyze other protein functions from sequences.
\end{abstract}

\footnotetext{
* Correspondence: syho@mail.nctu.edu.tw

${ }^{1}$ Department of Biological Science and Technology, National Chiao Tung

University, Hsinchu, Taiwan

Full list of author information is available at the end of the article
} 


\section{Background}

DNA-binding domains/proteins are functional proteins in a cell, which plays a vital role in various essential biological activities, such as DNA transcription, replication, packaging, repair and rearrangement [1]. In past, numerous computational methods predict DNA-binding domains/proteins using informative features from protein structure and amino acid sequence. The structurebased prediction methods such as [2,3] are accurate, but not capable for high throughput annotation. In this study, the sequence-based prediction methods for DNAbinding domains/proteins are investigated.

The computational methods using support vector machine (SVM) in conjunction with evolutionary information of amino acid sequence in terms of their position-specific scoring matrices (PSSMs) for predicting DNA-binding sites were successfully developed [4]. Several methods of using machine learning approaches were developed to predict DNA-binding domains/proteins from given sequences of variable lengths [5-9], shown in Table 1.

Due to different design aims and data sets used, it is difficult to assess which feature type is the most informative cooperated with SVM by comparing with prediction accuracies only. The PSSM profile was first used with SVM to successfully predict DNA-binding domains and proteins from query amino acid sequences in the method PSSM-400 [7]. The PSSM is an effective feature type of representing DNA-binding sequences, but its ability of interpretability is not satisfactory enough in analyzing the binding mechanism. Besides PSSMs, physicochemical properties with characteristics of high interpretability were commonly used $[5,6,8,9]$. Some issues are concerned in designing prediction methods, described below.

1) Selection of physicochemical properties. Generally, effective physicochemical properties of amino acids are selected as prediction features by using known properties of DNA-binding mechanism and binding knowledge $[5,6,8,9]$. However, it is desirable to explore undiscovered properties by machine learning approaches to further advance prediction accuracy and understand the binding mechanism.

2) Representation of sequences. How to effectively represent sequences of variable lengths as a feature vector using physicochemical properties plays an important role in advancing prediction accuracy. The pseudoamino acid composition is an efficient representation method of coupling physicochemical properties, which was used to represent a sequence as a 40-dimensional feature vector for discriminating DNA-binding from non-binding proteins [5]. The combined descriptor was proposed using amino acid composition and a series of associated physicochemical properties to form a 132dimensional feature vector [9]. The conjoint triad descriptor of using a 343-dimensional feature vector was proposed that 20 amino acids were clustered into seven classes according to their dipoles and volumes of side chains [8].

3) Values of amino acids for specific physicochemical properties. The AAindex database [10,11] collected 531 physicochemical and biochemical properties (ignoring 13 properties without available values) with corresponding values of amino acids. How to select an effective feature vector of 20 amino acid values to represent a given property by using experience of designers is not straightforward. Recently, some computational methods of predicting protein functions were successfully developed by mining informative physicochemical properties with their corresponding values from AAindex $[12,13]$.

4) Tradeoffs between prediction accuracy and knowledge acquisition. Besides pursuit of high prediction accuracy, discovering potential properties to further understand the binding mechanism should be also taken into account. Although ensemble classifiers with hybrid feature types and boosting techniques are commonly used to improve prediction performance, it is more desirable to find effective, interpretable physicochemical properties with strong discrimination abilities under using a simple SVM classifier.

This study presents a troublesome problem in using the AAindex database and proposes an effective method

Table 1 Related works of predicting DNA-binding domains/proteins from sequences

\begin{tabular}{|c|c|c|c|c|c|c|}
\hline Reference & Sequence type & Identity & Feature number & Representation & Feature type & Classifier \\
\hline Shao et al. 2009 [8] & protein & $25 \%$ & 343 & Seven class Conjoint triad & PCP & SVM \\
\hline Fang et al. 2008 [6] & protein & $35 \%$ & 40 & Pseudo-AA composition & PCP & SVM \\
\hline Yu et al. 2006 [9] & protein & $25 \%$ & 132 & Combined descriptors & PCP & SVM \\
\hline Cai et al 2003 [5] & protein & $40 \%$ & 40 & Pseudo-AA composition & PCP & SVM \\
\hline Kumar et al. 2007 [7] & domain and protein & $25 \%$ & 400 & PSSM & PSSM & SVM \\
\hline Auto-IDPCPs & domain and protein & $25 \%$ & $m^{*}$ & Mean value of sequence\# & PCP & SVM \\
\hline
\end{tabular}

PCP: physicochemical property and biochemical property.

*: a small number $(<30)$ of feature vectors selected from 531 vectors.

\#: The averaged value of amino acids in a sequence for one property. 
to solve. We found that some different physicochemical properties have similar vectors of representing 20 amino acids and some closely related physicochemical properties have dissimilar vectors. For example, several dissimilar vectors in AAindex are available in coupling the hydrophobicity property to represent a sequence and their corresponding performance is significantly different. Similarly, if a different property with a similar vector replaces the known one without significantly degrading prediction performance, it means that the replaced property may be also relative to the binding mechanism from the viewpoint of machine learning. The detailed explanation by using a real quantization example is described below.

Figure 1 shows an illustration example. The 402 properties in AAindex were classified into six groups according to their biological meanings classified by Tomii and Kanehisa [11], as shown in Fig. 2 obtained from [10]. According to the numerical indices representing 531 properties of amino acids, we clustered them into 20 clusters by using a fuzzy c-means algorithm based on their Euclidean distances between two indices [14]. The properties $\mathrm{H} 88$ and $\mathrm{A} 392$ are two different properties belonging to the same cluster 7 , but their distance of 0.0178 is relatively small. On the other hand, H88 and H178 belonging to the same group, named Hydrophobicity in AAindex, have a large distance of 0.0877 belonging to clusters 7 and 18, respectively. Although H88 and H151 (used in [5]) are in the same group Hydrophobicity and the same cluster 7, their distance 0.0299 is also larger than that between H88 and A392.

For the aim of designing accurate prediction methods, the major concern is to identify feature vectors with high discrimination abilities for classifying positive and negative samples. This task can be done well for computational methods by using an optimization approach to feature selection from a large set of candidate features. If the feature vectors were identified by predetermined properties based on prior knowledge, the selected vectors of representing amino acids may be not the best. Considering the other aim of discovering potential properties to further look insight the binding mechanism, we propose a systematic, optimization approach (named Auto-IDPCPs) to automatically identify a set of feature vectors and analyze the feature vectors to find properties of affecting the DNA-binding mechanism.

The proposed approach Auto-IDPCPs can identify a small number $m$ of feature vectors cooperated with a single SVM classifier, and discover the related hydrophobicity properties with comparable performance, compared with the PSSM feature. Auto-IDPCPs would help designers to investigate informative physicochemical and biochemical properties by considering both prediction accuracy and analysis of binding mechanism simultaneously. Auto-IDPCPs can be also applicable to predict and analyze other protein functions from sequences.

\section{Methods}

The flowchart of the proposed approach Auto-IDPCPs is shown in Fig. 3. The input of the method comprises the AAindex database and three data sets, including DNA-binding domains and sequences, and one independent test data set. The output has two parts: 1) a predictor for DNA-binding domains/proteins with a set of $m$ informative feature vectors and the parameter setting of SVM by using an efficient feature selection algorithm IBCGA, and 2) an analyzer with a set of promising physicochemical and biochemical properties in the AAindex database for analyzing the DNA-binding mechanism.

\section{Data sets}

To evaluate effectiveness of the identified physicochemical properties by comparing with the famous PSSM features, we used the benchmark data sets used in the PSSM-400 method [7], as shown in Table 2.

\begin{tabular}{|c|c|c|c|}
\hline & Group ID & AAindex ID & Description \\
\hline & H88 & FAUJ880111 & Positive charge (Fauchere et al.1988) \\
\hline & H178 & MEEJ800101 & $\begin{array}{l}\text { Retention coefficient in HPLC, pH7.4 } \\
\text { (Meek, 1980) }\end{array}$ \\
\hline \begin{tabular}{c|c} 
Cluster 7 \\
0.0877 & $0.091 \% / 0.0801$
\end{tabular} & H151 & KYTJ820101 & $\begin{array}{l}\text { Hydropathy index (Kyte-Doolittle, } \\
\text { 1982) }\end{array}$ \\
\hline Cluster $18 \quad \mathrm{H} 178$ & A392 & WOLS 870103 & $\begin{array}{l}\text { Principal property value } z 3 \text { (Wold et } \\
\text { al., 1987) }\end{array}$ \\
\hline
\end{tabular}

Figure 1 An illustration example. The properties H88 and A392 are two different properties but their distance 0.0178 is relatively small. On the other hand, $\mathrm{H} 88$ and $\mathrm{H} 178$ belonging to the same group Hydrophobicity in AAindex have a large distance 0.0877 . H88 and H151 in the same group have a larger distance 0.0299 than that between H88 and A392. 


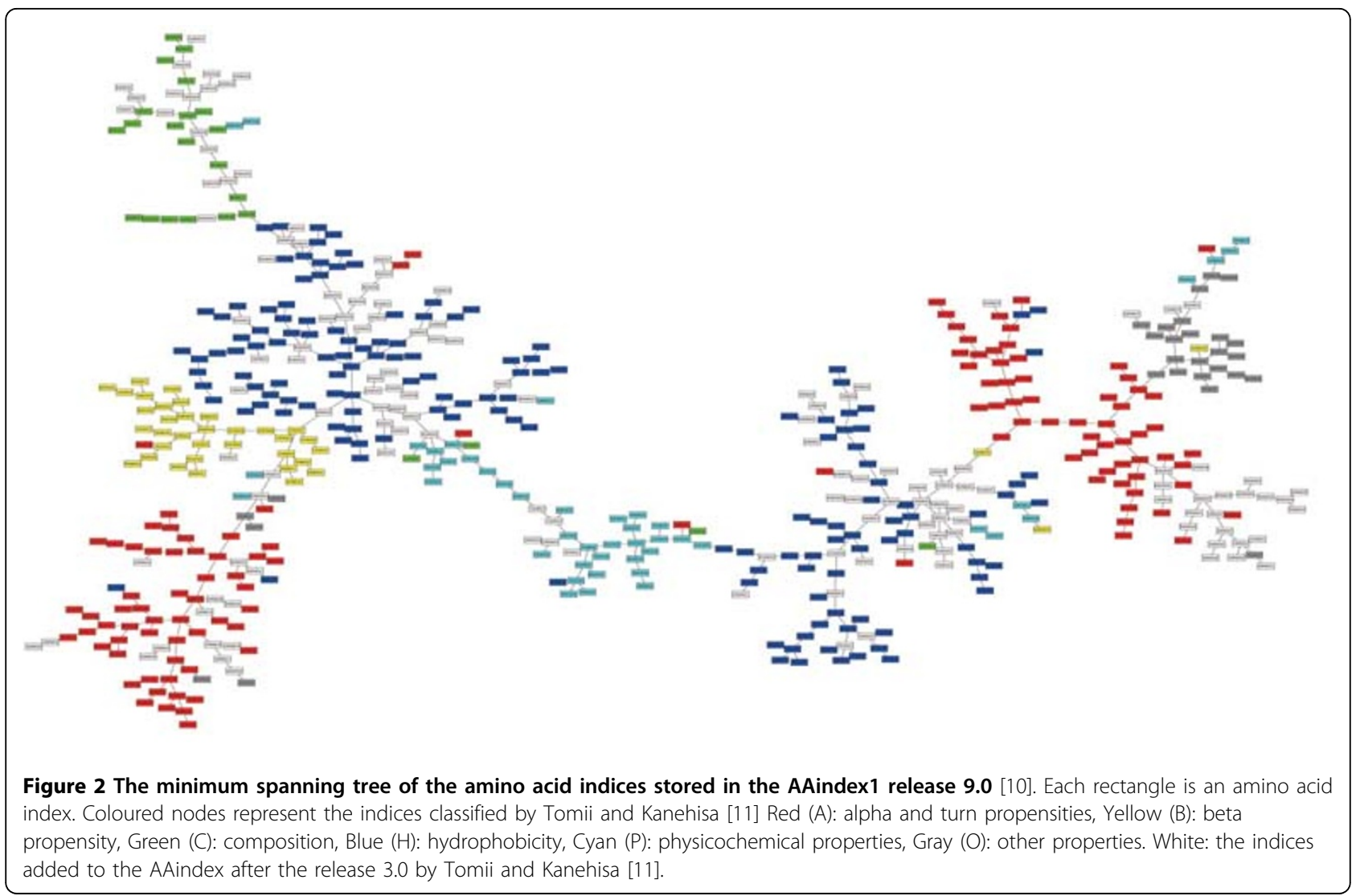

The data set DNAset has 146 non-redundant DNAbinding domains (or protein chains) and 250 nonbinding domains. No two domains have similarity more than $25 \%$. The data set DNAaset consists of 1153 DNA-binding proteins and 1153 non-binding proteins. No two proteins have similarity more than $25 \%$. An independent data set DNAiset is additionally used, having 92 DNA-binding domains and 100 nonDNA-binding proteins [7].

\section{Feature vector representation}

All the domains/sequences have a variable length $l$. A sequence forms an $l$-dimensional profile where the value of each amino acid is obtained from the AAindex database for encoding a specific physicochemical property. The $l$-dimensional profiles are transformed into vectors with the same constant length $L$ for utilizing SVM. The transformation can be any known effective representation provided that the $\mathrm{L}$ features can

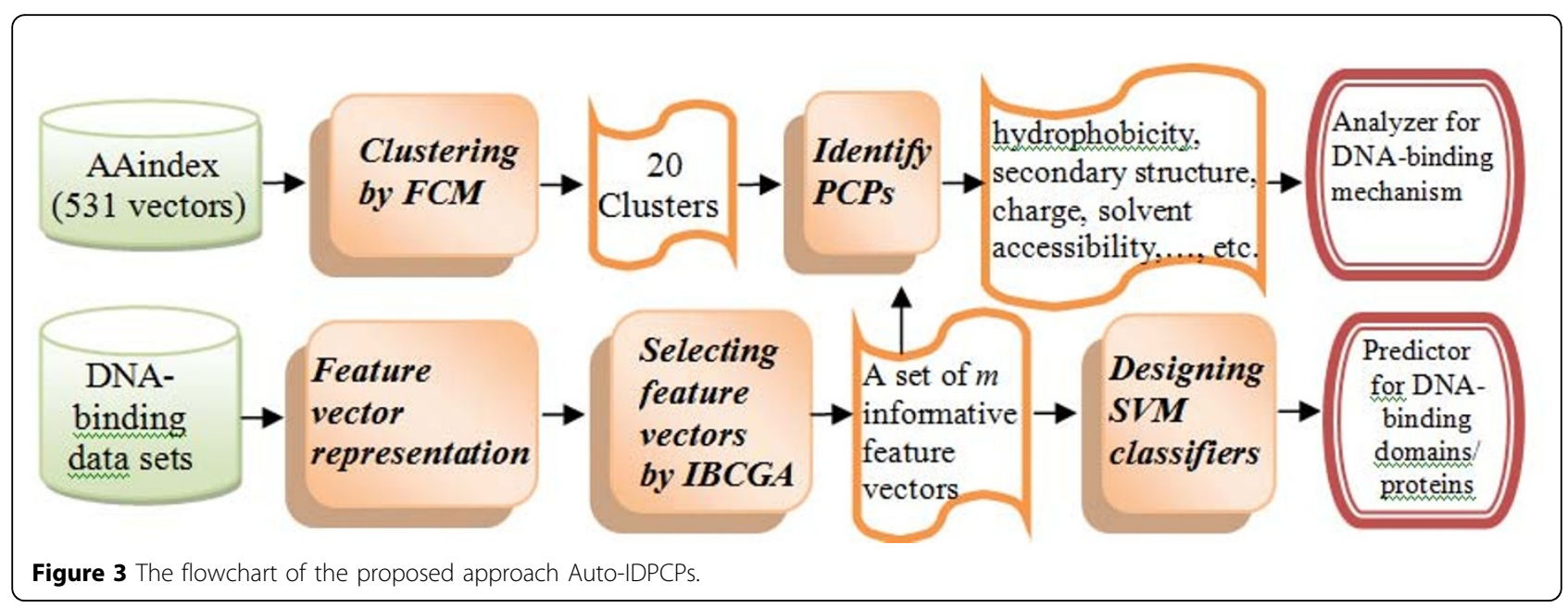


Table 2 The statistic data of the three data sets

\begin{tabular}{lccc}
\hline Datasets & Sequence & $\begin{array}{c}\text { No. of DNA- } \\
\text { binding }\end{array}$ & $\begin{array}{c}\text { No. of non-DNA- } \\
\text { binding }\end{array}$ \\
\hline DNAset & domain & 146 & 250 \\
DNAaset & protein & 1153 & 1153 \\
DNAiset & $\begin{array}{c}\text { domain / } \\
\text { protein }\end{array}$ & 92 & 100 \\
\hline
\end{tabular}

effectively classify the $l$-dimensional profiles of positive and negative sequences. The simplest feature is the mean of the profile that $\mathrm{L}=1[12,13]$. Therefore, the sequences with $m$ properties are represented as an $m$ dimensional feature vectors. Finally, all values of the feature vectors are normalized into $[-1,1]$ to apply SVM.

\section{Feature selection by IBCGA}

Selecting a minimal number of informative features while maximizing prediction accuracy is a bi-objective $0 / 1$ combinatorial optimization problem. An efficient inheritable bi-objective combinatorial genetic algorithm IBCGA [15] bases on an intelligent genetic algorithm IGA [16] is utilized to solve this optimization problem. IGA based on orthogonal experimental design uses a divide-and-conquer strategy and a systematic reasoning method instead of the conventional generate-and-go method to efficiently solve the combinatorial optimization problem $C(n, m)$ having a huge search space of size $n ! /(m !(n-m) !))$, where $n=531$ in this study. IBCGA can efficiently search the space of $C(n, r \pm 1)$ by inheriting a good solution in the space of $C(n, r)$ [15]. Therefore, IBCGA can economically obtain a complete set of high-quality solutions in a single run.

The chromosome encoding scheme of IGA consists of $n$ binary IGA-genes $g_{i}$ for selecting informative features (physicochemical properties) and two 4-bit IGA-genes for encoding $\gamma$ and $C$ of SVM parameters, where $\gamma \in\left\{2^{-}\right.$ $\left.7,2^{-6} \ldots, 2^{8}\right\}$ and $C \in\left\{2^{-7}, 2^{-6} \ldots, 2^{8}\right\}$ [12]. The $i^{\text {th }}$ feature is used in the SVM classifier if $g_{i}=1$; otherwise, the $i^{\text {th }}$ feature is excluded $\left(g_{i}=0\right)$. The performance of selected properties associated with the parameter values of SVM is measured by five-fold cross-validation (5-CV) for comparing with the method PSSM-400 [7]. IBCGA with the fitness function $f(\mathrm{X})$ of $5-\mathrm{CV}$ prediction accuracy can simultaneously obtain a set of solutions, $\mathrm{X}_{r}$, where $r=r_{\text {start }}, r_{\text {start }}+1, \ldots, r_{\text {end }}$ in a single run. In this study, the parameter settings $r_{\text {start }}=10, r_{\text {end }}=30, N_{\text {pop }}=50$, $p_{c}=0.8$, and $p_{m}=0.05$. The output contains a set of $m$ selected properties from AAindex and an SVM classifier with associated parameter settings $\gamma$ and $C$. The customized IBCGA algorithm used in Auto-IDPCPs is given below.
Step 1: (Initiation) Randomly generate an initial population of $N_{\text {pop }}$ individuals. All the $n$ binary genes have $r$ 1's and $n-r$ 0's where $r=r_{\text {start }}$.

Step 2: (Evaluation) Evaluate the fitness values of all individuals using $f(\mathrm{X})$.

Step 3: (Selection) Use the traditional tournament selection that selects the winner from two randomly selected individuals to form a mating pool.

Step 4: (Crossover) Select $p_{c} \cdot N_{\text {pop }}$ parents from the mating pool to perform orthogonal array crossover on the selected pairs of parents where $p_{c}$ is the crossover probability.

Step 5: (Mutation) Apply the mutation operator to the randomly selected $p_{m} \cdot N_{\text {pop }}$ individuals in the new population where $p_{m}$ is the mutation probability. To prevent the best fitness value from deteriorating, mutation is not applied to the best individual.

Step 6: (Termination test) If the stopping condition for obtaining the solutions $\mathrm{X}_{r}$ is satisfied, output the best individual as $\mathrm{X}_{r}$. Otherwise, go to Step 2 .

Step 7: (Inheritance) If $\mathrm{r}<r_{\text {end }}$, randomly change one bit in the binary genes for each individual from 0 to 1 ; increase the number $r$ by one, and go to Step 2 .

Step 8: (System uncertainty) Perform Steps 1-7 for $R$ (=30 in this study) independent runs and obtain the best one of $R$ solutions. The best solution can be determined by considering the most accurate one $S_{\mathrm{a}}$ with the highest prediction accuracy or the robust one $S_{\mathrm{r}}$ with the highest score, where the scores $S_{t}, t=1, \ldots, R$ are derived using the following procedure Appscore.

The procedure Appscore is described as follows:

Step 1: Calculate the appearance frequency $\mathrm{f}\left(p_{i}\right)$ of each feature $p_{i}$ from all features in the $R$ sets, where $i=1, \ldots, m_{t}$. There are $m_{\hat{A} \neg t}$ features in the $t$-th set.

Step 2: Calculate score $S_{t} t=1, \ldots, R$ for each of $R$ solutions:

$$
S_{t}=\left(\sum_{i=1}^{m_{t}} \mathrm{f}\left(p_{i}\right)\right) / m_{t}
$$

\section{Clustering properties by fuzzy c-means}

The physicochemical properties can be classified into six groups according to their biological meanings [11]. From the viewpoint of machine learning, two properties are similar if the distance between their feature vectors is small. To identify informative physicochemical properties and obtain effective feature vectors with strong discriminative abilities, we cluster the 531 vectors of physicochemical properties into 20 clusters using a fuzzy c-means (FCM) method [14].

A feature vector of amino acids is a set of 20 numerical values representing a physicochemical property of amino acids. To apply the FCM method, all data were normalized in such a way that every physicochemical 
Table 3 The 20 clusters and their corresponding physicochemical and biochemical properties in the AAindex database Cluster No. The label of 531 physicochemical and biochemical properties

\begin{tabular}{|c|c|c|}
\hline$C_{1}$ & 2 & P: 118 O: 156 \\
\hline $\mathrm{C}_{2}$ & 2 & P: 504505 \\
\hline$C_{3}$ & 6 & H: 1011446447448449 \\
\hline $\mathrm{C}_{4}$ & 3 & P: 9112150 \\
\hline $\mathrm{C}_{5}$ & 4 & C: 116 H: 34127 P: 117 \\
\hline $\mathrm{C}_{6}$ & 6 & A: 313 H: 129145364 P: 177 O: 312 \\
\hline $\mathrm{C}_{7}$ & 147 & 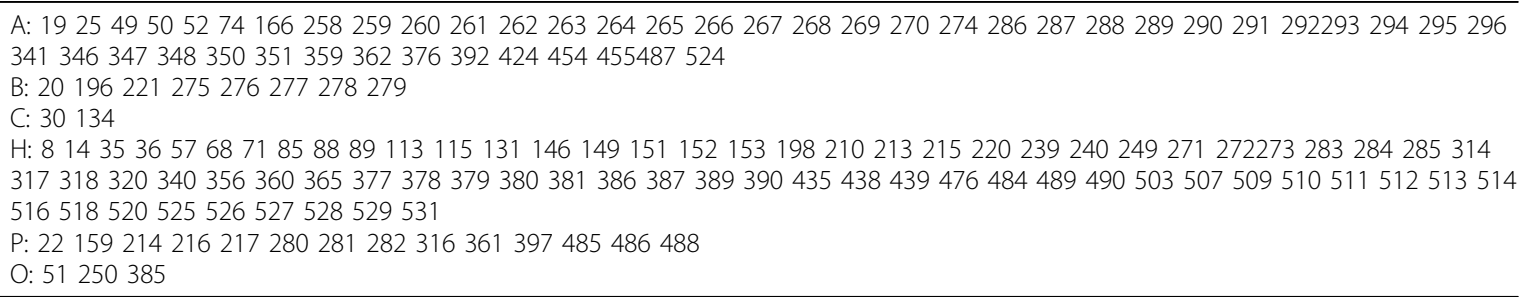 \\
\hline $\mathrm{C}_{8}$ & 3 & P: 65135517 \\
\hline $\mathrm{C}_{9}$ & 132 & $\begin{array}{l}\text { A: } 57243740444748536293104105107121122124162165176188227228229230235236237238255303309334335 \\
337338345367369375413417418420428429430432433436498 \\
\text { B: } 106 \\
\text { C: } 31144 \\
\text { H: } 6418687929495110114125142143182183297298299300302325326327354355357371373384403404405406 \\
407408421422425427431453482491492493494496497499506530 \\
\text { P: } 152859219383391 \\
\text { O: } 21274379123126173174175251301305308322323324336370372374409419\end{array}$ \\
\hline$C_{10}$ & 123 & $\begin{array}{l}\text { A: } 38426091979899100119138140160163171186223224231253256307311328330331333339342349363366410 \\
411412414415416426 \\
\text { B: } 39454661101102103120139141161164167168169172187218225226232234254257310343344368445495 \\
\text { C: } 190304 \\
\text { H: } 2342354555658666769707677108130132133192194201211212233243315321329332352358398477478480 \\
481508515519521522523 \\
\text { P: } 2629788296154157473479 \\
\text { O: } 306437\end{array}$ \\
\hline$C_{11}$ & 6 & P: 3272109353474475 \\
\hline $\mathrm{C}_{12}$ & 2 & $\mathrm{H}: 128483$ \\
\hline $\mathrm{C}_{13}$ & 1 & C: 137 \\
\hline$C_{14}$ & 15 & $\begin{array}{l}\text { H: } 170241244245246393395396400402423444 \\
\text { P: } 399502 \\
\text { O: } 84\end{array}$ \\
\hline $\mathrm{C}_{15}$ & 1 & $\mathrm{H}: 73$ \\
\hline$C_{16}$ & 43 & $\begin{array}{l}\text { A: } 18 \\
\text { C: } 64136189191193195197199200202203204205206207208209440441442443456457458459460461462463464 \\
465466467468469470471472 \\
\text { H: } 147148222252\end{array}$ \\
\hline $\mathrm{C}_{17}$ & 3 & $\mathrm{H}: 450451452$ \\
\hline $\mathrm{C}_{18}$ & 28 & $\begin{array}{l}\text { A: } 1690 \\
\text { H: } 1213111178179180181185242247248382388394401500501 \\
\text { P: } 11763808183158434 \\
\text { O: } 75\end{array}$ \\
\hline$C_{19}$ & 2 & $\begin{array}{l}\mathrm{H}: 184 \\
\mathrm{O}: 155\end{array}$ \\
\hline $\mathrm{C}_{20}$ & 2 & P: 33319 \\
\hline
\end{tabular}

A: Alpha and turn propensities. B: Beta propensity. C: Composition. H: Hydrophobicity. P: Physicochemical properties. O: Other properties. 
property had an averaged profile value of zero and a standard deviation equal to 1 . The FCM method has an objective functional of the form [14]:

$$
\mathrm{J}(\mathrm{X} ; \mathrm{U}, \mathrm{v})=\sum_{i=1}^{K} \sum_{j=1}^{n} u_{i j}^{s} d^{2}\left(v_{i}, x_{j}\right)
$$

where $n=531$ is the number of data vectors, $K$ is the number of clusters to be found, $u_{i j} \in[0,1]$ is the membership degree of $j^{\text {th }}$ data vector $x_{j}$ in the $i^{\text {th }}$ cluster, the $i^{\text {th }}$ cluster represented by the cluster prototype $v_{i}$, $s \in[1, \infty)$ is a weighting exponent called the fuzzifier and $d\left(v_{i}, x_{j}\right)$ is the distance of $x_{j}$ from the cluster prototype $v_{i}$. Dembélé and Kastner [17] suggested the parameters setting $s=1.12$ and $K=20$ clusters, adopted in this study.

\section{Identifying physicochemical properties}

It is not easy to discover related physicochemical properties for analyzing DNA-binding mechanism by computational methods using relatively small-size data sets. Therefore, we present a hybrid method by combining evidences from the viewpoints of both machine learning and biological meanings. Auto-IDPCPs identifies $m$ properties belong to $c$ out of 20 clusters. We examine each property P1 of the 531 properties and each property P2 of the identified $m$ properties. If they satisfy the following two criteria, P1 is a promising property to be further investigated: 1 ) the distance between the feature vectors of P1 and P2 is small, and 2) P2 is replaced with $\mathrm{P} 1$ one at a time and the prediction accuracy is not significantly decreased.

Only 402 of 531 properties were classified into six groups [11], (A): Alpha and turn propensities, (B): Beta propensity, $(\mathrm{C})$ : Composition, $(\mathrm{H})$ : Hydrophobicity, $(\mathrm{P})$ : Physicochemical properties, and $(\mathrm{O})$ : Other properties. We classified the other 129 properties into the six groups according to their distances of vectors using a nearest-neighbour rule. The 531 featured IDs and their corresponding AAindex IDs are listed in Table S1 (see Additional File 1). The 20 clusters and their corresponding physicochemical and biochemical properties belonging to six groups in the AAindex database are given in Table 3. The statistical result of the 531 physicochemical properties distributed upon the 20 clusters and six groups is given in Table 4. The large clusters 7, 9 and 10 have properties belonging to six groups. The valuable information in Tables 3 and 4 integrating classification results of both biological meanings and similarity measure is very helpful in identifying informative properties. The statistical results of property distribution in the six groups for 531 and 402 amino acid indices are given in Fig. 4. The largest group is Hydrophobicity having 149 indices and the second largest group is Alpha and turn
Table 4 The statistical result of the 531 physicochemical properties distributed upon the $\mathbf{2 0}$ clusters and six groups

\begin{tabular}{|c|c|c|c|c|c|c|c|}
\hline Cluster & $A$ & B & $C$ & $\mathrm{H}$ & $P$ & 0 & TOTAL \\
\hline$C_{1}$ & & & & & 1 & 1 & 2 \\
\hline$C_{2}$ & & & & & 2 & & 2 \\
\hline$C_{3}$ & & & & 6 & & & 6 \\
\hline$C_{4}$ & & & & & 3 & & 3 \\
\hline$C_{5}$ & & & 1 & 2 & 1 & & 4 \\
\hline$C_{6}$ & 1 & & & 3 & 1 & 1 & 6 \\
\hline$C_{7}$ & 47 & 7 & 2 & 74 & 14 & 3 & 147 \\
\hline$C_{8}$ & & & & & 3 & & 3 \\
\hline$C_{9}$ & 51 & 1 & 3 & 50 & 6 & 21 & 132 \\
\hline$C_{10}$ & 38 & 30 & 2 & 42 & 9 & 2 & 123 \\
\hline$C_{11}$ & & & & & 6 & & 6 \\
\hline$C_{12}$ & & & & 2 & & & 2 \\
\hline$C_{13}$ & & & 1 & & & & 1 \\
\hline$C_{14}$ & & & & 12 & 2 & 1 & 15 \\
\hline$C_{15}$ & & & & 1 & & & 1 \\
\hline$C_{16}$ & 1 & & 38 & 4 & & & 43 \\
\hline$C_{17}$ & & & & 3 & & & 3 \\
\hline$C_{18}$ & 3 & & & 17 & 8 & & 28 \\
\hline$C_{19}$ & & & & 1 & & 1 & 2 \\
\hline$C_{20}$ & & & & & 2 & & 2 \\
\hline TOTAL & 141 & 38 & 47 & 217 & 58 & 30 & 531 \\
\hline RATE & 0.266 & 0.072 & 0.089 & 0.409 & 0.109 & 0.056 & \\
\hline
\end{tabular}

A: Alpha and turn propensities. B: Beta propensity. C: Composition. H: Hydrophobicity. P: Physicochemical properties. O: Other properties.

propensities having 118 indices (Fig. 4b). The sizes of the other four groups are relatively small.

\section{Results}

Identified properties by IBCGA

The statistical result of $S_{t}$ in selecting property sets from $R=30$ independent runs on DNAset and DNAaset is given in Fig. 5. The $18^{\text {th }}$ and $6^{\text {th }}$ runs having the highest scores for DNAset and DNAaset respectively are selected, and their prediction accuracies for various numbers of selected features are given in Fig. 6. The robust solutions $\mathrm{S}_{18}$ and $\mathrm{S}_{6}$ having $m=22$ and 28 features with accuracies of $87.12 \%$ and $75.50 \%$ for DNAset and DNAaset, respectively, are given in Tables 5 and 6 , where the AAindex identity numbers and their property descriptions are provided.

An efficient way to study effects of several factors simultaneously is to utilize orthogonal experimental design used in IGA [16]. The main effect difference (MED) of orthogonal experimental design can estimate effects of individual features according to the value of MED [12,16]. The most effective property has the largest value of MED. The $m$ properties ranked by using MED are shown in Fig. 7. The properties of rank 1 are identity numbers H86 (FAUJ880109, Number of hydrogen bond donors) and B39 (CHOP780202, Normalized 


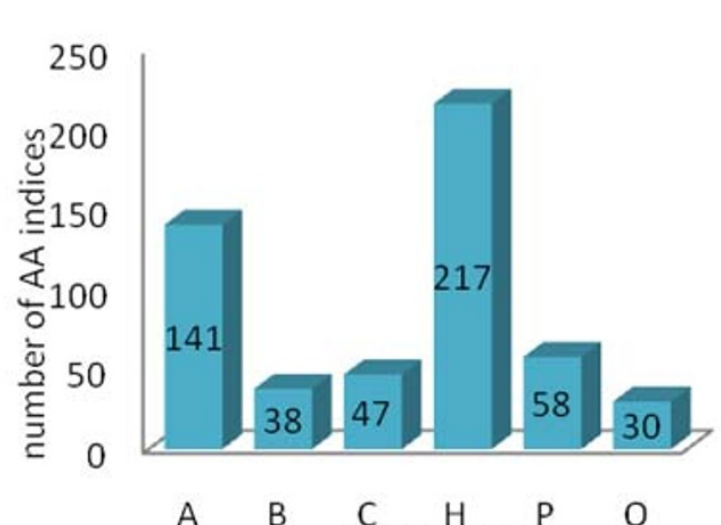

A B $\underset{\text { sixgroups }}{\mathrm{C}} \mathrm{P} \quad \mathrm{O}$

(a)

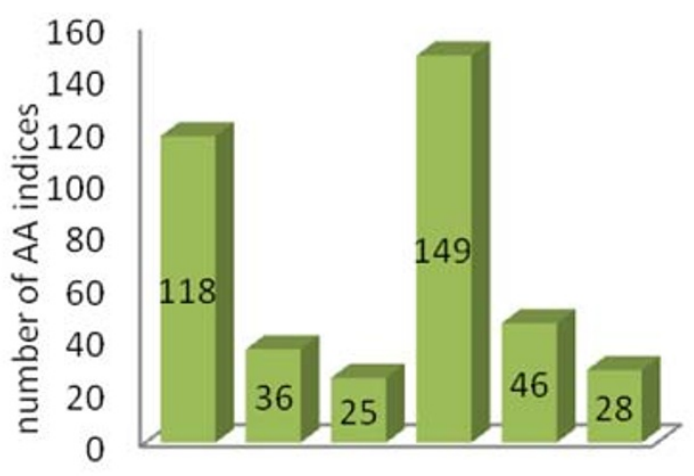

A B $\underset{\text { sixgroups }}{\mathrm{C}} \underset{\mathrm{H}}{\mathrm{H}} \mathrm{O}$

(b)

Figure 4 The statistical results of property distribution in the six groups (a) 531 amino acid indices (b) 402 amino acid indices.
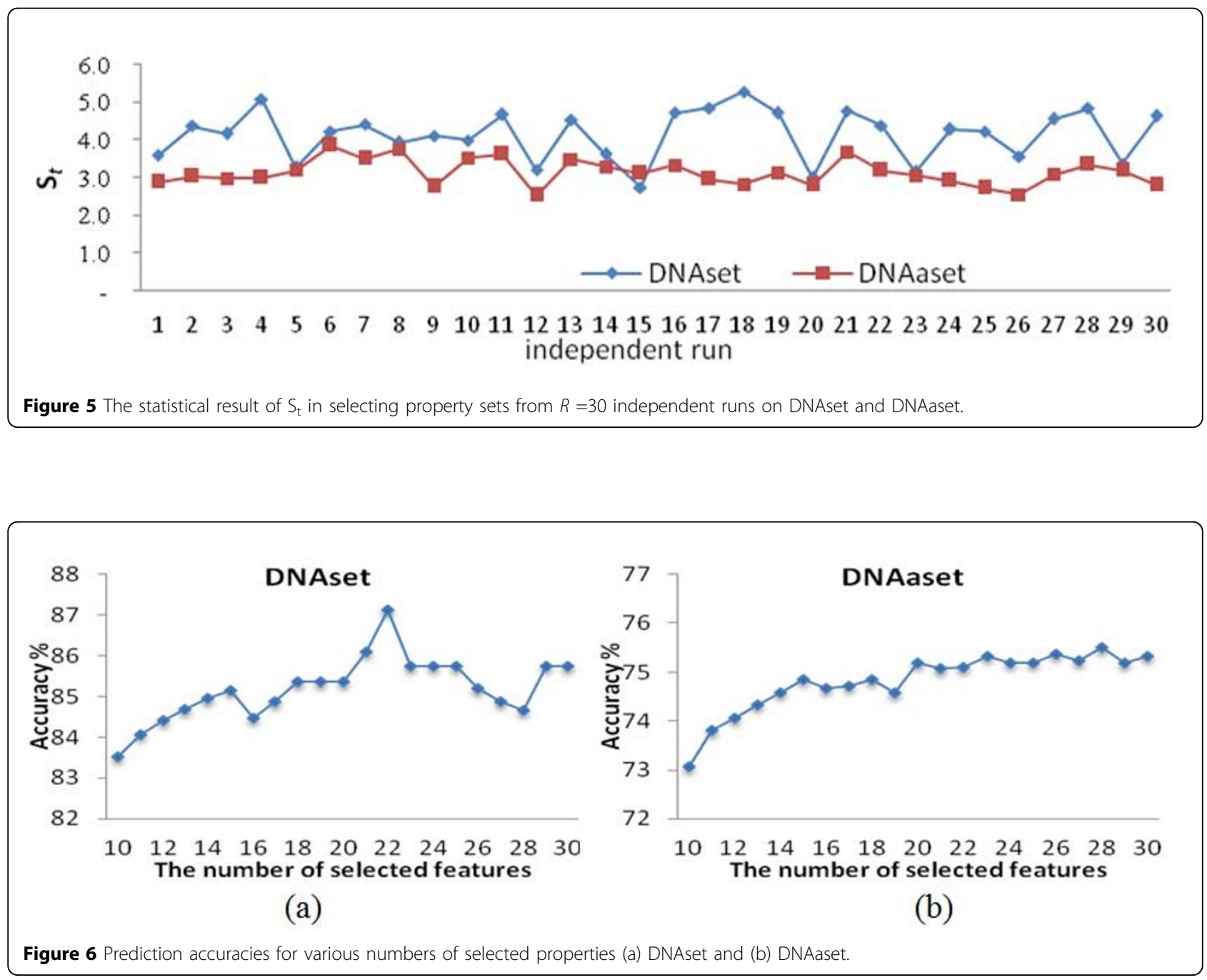
Table 5 The robust solution $S_{18}$ with a set of $m=22$ features for DNAset

\begin{tabular}{|c|c|c|}
\hline Feature ID & AAindex ID & Description \\
\hline 53 & CHOP780216 & Normalized frequency of the 2nd and 3rd residues in turn (Chou-Fasman, 1978b) \\
\hline 56 & $\mathrm{CIDH} 920103$ & Normalized hydrophobicity scales for alpha+beta-proteins (Cid et al., 1992) \\
\hline 64 & DAYM780101 & Amino acid composition (Dayhoff et al., 1978a) \\
\hline 86 & FAUJ880109 & Number of hydrogen bond donors (Fauchere et al., 1988) \\
\hline 91 & FINA770101 & Helix-coil equilibrium constant (Finkelstein-Ptitsyn, 1977) \\
\hline 188 & NAGK730103 & Normalized frequency of coil (Nagano, 1973) \\
\hline 202 & NAKH920101 & AA composition of CYT of single-spanning proteins (Nakashima-Nishikawa, 1992) \\
\hline 227 & PALJ810105 & Normalized frequency of turn from LG (Palau et al., 1981) \\
\hline 228 & PALJ810106 & Normalized frequency of turn from CF (Palau et al., 1981) \\
\hline 255 & PRAM900104 & Relative frequency in reverse-turn (Prabhakaran, 1990) \\
\hline 262 & QIAN880105 & Weights for alpha-helix at the window position of -2 (Qian-Sejnowski, 1988) \\
\hline 274 & QIAN880117 & Weights for beta-sheet at the window position of -3 (Qian-Sejnowski, 1988) \\
\hline 286 & QIAN880129 & Weights for coil at the window position of -4 (Qian-Sejnowski, 1988) \\
\hline 363 & SUEM840101 & Zimm-Bragg parameter s at 20 C (Sueki et al., 1984) \\
\hline 383 & WEBA780101 & RF value in high salt chromatography (Weber-Lacey, 1978) \\
\hline 388 & WOEC730101 & Polar requirement (Woese, 1973) \\
\hline 412 & AURR980110 & Normalized positional residue frequency at helix termini N5 (Aurora-Rose, 1998) \\
\hline 430 & MUNV940102 & Free energy in alpha-helical region (Munoz-Serrano, 1994) \\
\hline 434 & WIMW960101 & Free energies of transfer of AcWI-X-LL peptides from bilayer interface to water (Wimley-White, 1996) \\
\hline 443 & KUMS000104 & Distribution of amino acid residues in the alpha-helices in mesophilic proteins (Kumar et al., 2000) \\
\hline 486 & BASU050102 & $\begin{array}{l}\text { Interactivity scale obtained by maximizing the mean of correlation coefficient over single-domain globular proteins } \\
\text { (Bastolla et al., 2005) }\end{array}$ \\
\hline 513 & JACR890101 & Weights from the IFH scale (Jacobs-White, 1989) \\
\hline
\end{tabular}

frequency of beta-sheet) for DNAset and DNAaset, belonging to the groups Hydrophobicity and Beta propensity, respectively.

\section{Prediction performance evaluation}

To evaluate the effectiveness of the identified $m$ informative feature vectors (PCPs), we implemented the predictor using the same single classifier SVM with the feature types, amino acid composition (AAC) and PSSM [7]. Additionally, the selected PCPs combined with AAC and PSSM were also evaluated, as shown in Table 7. AAC is a 20-dimensional vector of amino acid composition. PSSM is the feature representation of 400 features. PCPs + AAC and PCPs +PSSM are two hybrid feature types.

Considering the DNA-binding domain data set DNAset, the set of $m=22$ informative properties (PCPs) identified by Auto-IDPCPs performs best where the robust solution $S_{r}$ with accuracy of $87.12 \%$ is used. The four compared methods AAC, PSSM, PCPs+AAC, and PCPs + PSSM have accuracies of $80.30 \%, 86.62 \%, 81.82 \%$ and $86.62 \%$, respectively. For the DNA-binding protein data set DNAaest, the method using PCPs with $m=28$ informative properties $(75.50 \%)$ is slightly better than the PSSM-400 (74.22\%). However, PCPs+PSSM can improve both PCPs and PSSM to the accuracy of $80.27 \%$. When the method using the trained SVM with PCPs was evaluated by the independent test data set DNAiset, the method using selected PCPs has the accuracy of $80.73 \%$ (=155/192), slightly worse than the method PSSM-400 with the accuracy of $82.81 \%(=159 / 192)$ [7].

A small, high-performance features set of size $c$ that one property is selected from each of the identified $\mathrm{C}$ clusters using the optimization algorithm IGA is given in Table 8 , where $\mathrm{c}=5$ and 8 for DNAset and DNAaset, respectively. The accuracies of the robust solutions using the c features with SVM are $83.59 \%$ and $73.24 \%$ for DNAset and DNAaset, respectively. The properties and their descriptions are given in Tables 9 and 10 .

The experimental results reveal that the identified small set of $m$ physicochemical properties with a simple sequence representation and a single SVM classifier is promising, compared with the PSSM feature type. However, the identified physicochemical properties are interpretable for further understanding the DNA-binding mechanism.

\section{Analyzing physicochemical properties for binding mechanism}

The selected features of the conducted 30 runs are very different in terms of feature ID from 531 properties. The appearance frequency of each identified cluster in 
Table 6 The robust solution $S_{6}$ with a set of $m=28$ features for DNAaset

\begin{tabular}{|c|c|c|}
\hline $\begin{array}{c}\text { Feature } \\
\text { ID }\end{array}$ & AAindex ID & Description \\
\hline 39 & CHOP780202 & Normalized frequency of beta-sheet (Chou-Fasman, 1978b) \\
\hline 56 & $\mathrm{CIDH} 920103$ & Normalized hydrophobicity scales for alpha+beta-proteins (Cid et al., 1992) \\
\hline 58 & $\mathrm{CIDH} 920105$ & Normalized average hydrophobicity scales (Cid et al., 1992) \\
\hline 86 & FAUJ880109 & Number of hydrogen bond donors (Fauchere et al., 1988) \\
\hline 88 & FAUJ880111 & Positive charge (Fauchere et al., 1988) \\
\hline 95 & FINA910104 & Helix termination parameter at posision j+1 (Finkelstein et al., 1991) \\
\hline 100 & GEIM800104 & Alpha-helix indices for alpha/beta-proteins (Geisow-Roberts, 1980) \\
\hline 102 & GEIM800106 & Beta-strand indices for beta-proteins (Geisow-Roberts, 1980) \\
\hline 139 & KANM800102 & Average relative probability of beta-sheet (Kanehisa-Tsong, 1980) \\
\hline 146 & KLEP840101 & Net charge (Klein et al., 1984) \\
\hline 147 & KRIW710101 & Side chain interaction parameter (Krigbaum-Rubin, 1971) \\
\hline 167 & LIFS790101 & Conformational preference for all beta-strands (Lifson-Sander, 1979) \\
\hline 178 & MEEJ800101 & Retention coefficient in HPLC, pH7.4 (Meek, 1980) \\
\hline 214 & OOBM770102 & Short and medium range non-bonded energy per atom (Oobatake-Ooi, 1977) \\
\hline 229 & PALJ810107 & Normalized frequency of alpha-helix in all-alpha class (Palau et al., 1981) \\
\hline 280 & QIAN880123 & Weights for beta-sheet at the window position of 3 (Qian-Sejnowski, 1988) \\
\hline 299 & RACS770103 & Side chain orientational preference (Rackovsky-Scheraga, 1977) \\
\hline 321 & RADA880108 & Mean polarity (Radzicka-Wolfenden, 1988) \\
\hline 356 & ROSM880102 & Side chain hydropathy, corrected for solvation (Roseman, 1988) \\
\hline 365 & SWER830101 & Optimal matching hydrophobicity (Sweet-Eisenberg, 1983) \\
\hline 399 & ZIMJ680102 & Bulkiness (Zimmerman et al., 1968) \\
\hline 401 & ZIMJ680104 & Isoelectric point (Zimmerman et al., 1968) \\
\hline 422 & AURR980120 & Normalized positional residue frequency at helix termini C4' (Aurora-Rose, 1998) \\
\hline 431 & MUNV940103 & Free energy in beta-strand conformation (Munoz-Serrano, 1994) \\
\hline 449 & NADH010104 & Hydropathy scale based on self-information values in the two-state model (20\% accessibility) (Naderi-Manesh et al., 2001) \\
\hline 451 & NADH010106 & Hydropathy scale based on self-information values in the two-state model (36\% accessibility) (Naderi-Manesh et al., 2001) \\
\hline 512 & GUYH850105 & Apparent partition energies calculated from Chothia index (Guy, 1985) \\
\hline 528 & MIYS990104 & Optimized relative partition energies - method C (Miyazawa-Jernigan, 1999) \\
\hline
\end{tabular}

the 30 runs is shown in Fig. 8. From the statistic result, the clusters $7,9,10,16$ and 18 with very high selection frequencies are more informative for predicting DNAbinding domains and proteins. The selected clusters of the 30 runs are very similar in terms of cluster ID from 20 clusters. The $m=22$ properties (Table 5) belong to five clusters which are the same with the five clusters 7 , 9, 10, 16 and 18. For predicting DNA-binding proteins, the $m=28$ properties (Table 6) belong to eight clusters, which are the same five clusters and additional three clusters 3,14 and 17 . The results reveal that the identified features of the robust solution $S_{\mathrm{r}}$ belong to

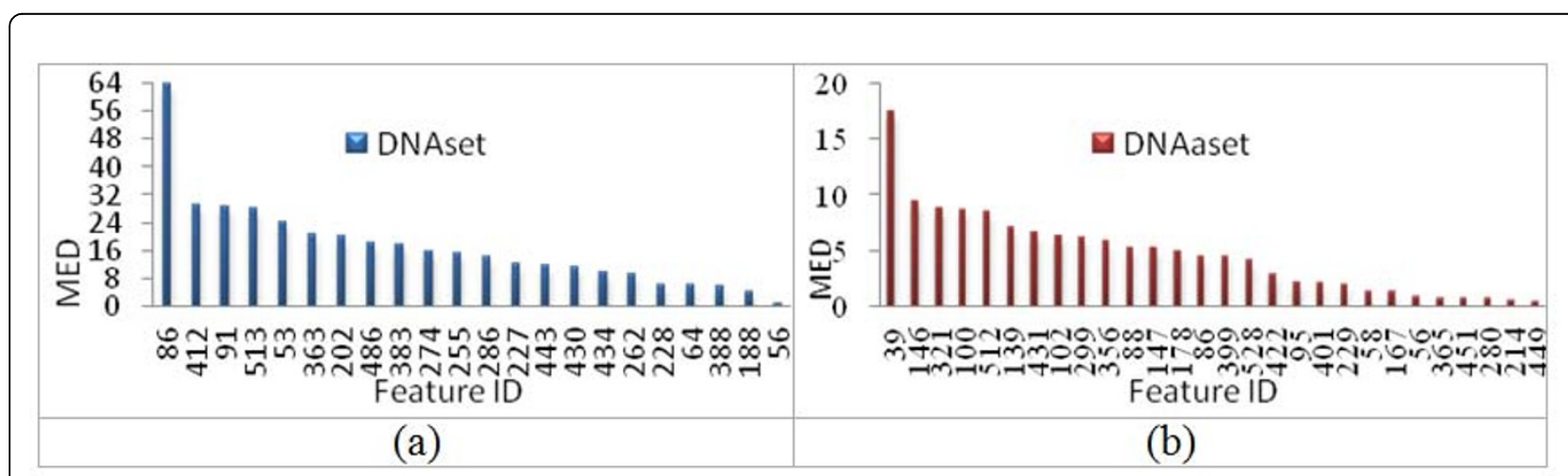

Figure 7 The $m$ properties ranked by using main effect difference (MED) (a) $m=22$ for DNAset and (b) $m=28$ for DNAaset. 
Table 7 The overall accuracies (\%) of 5-CV for three feature types and two hybrid feature types with SVM

\begin{tabular}{lllllllllll}
\hline Dataset & & Sen. & Spe. & MCC & PCPs & AAC & PSSM* & $\begin{array}{c}\text { PCPs } \\
\text { +AAC }\end{array}$ & $\begin{array}{c}\text { PCPs } \\
\text { +PSSM }\end{array}$ \\
\hline DNAset & $S_{a}$ & 88.89 & 91.20 & 0.76 & 88.89 & 80.30 & 86.62 & 81.57 & 83.59 \\
\cline { 2 - 5 } \cline { 7 - 8 } & $S_{r}$ & 82.19 & 90.00 & 0.53 & 87.12 & & & 81.82 & 86.62 \\
\hline DNAaest & $S_{a}$ & 82.74 & 70.08 & 0.72 & 76.41 & 72.46 & 74.22 & 74.20 & 79.88 \\
\cline { 2 - 4 } \cline { 6 - 7 } & $S_{r}$ & 81.96 & 69.04 & 0.51 & 75.50 & & & 73.59 & 80.27 \\
\hline
\end{tabular}

$S_{a}$ : accurate solution, $S_{r}$ : robust solution, Sen.: sensitivity, Spe.: specificity, MCC: Matthew's correlation coefficient, PCPs: the $m$ informative properties, PSSM*: obtained from [7].

informative clusters. Therefore, the informative clusters can provide informative properties to be further investigated if these properties were not selected by the feature selection method.

An illustration example for exploring promising properties is given in Fig. 9. The both feature sets S1 (H88, H86, H67, C209, H178) and S2 (A392, A303, A307, C440, H178) are selected for predicting DNAbinding domains in DNAset that one property is selected from one of five clusters $7,9,10,16$ and 18 . The identified properties H88 and A392 belong to the
Hydrophobicity, and Alpha and turn propensities groups respectively, but they belong to the same cluster 7 with a relatively small distance 0.0178 . The prediction accuracy of S3 by replacing $\mathrm{H} 88$ with $\mathrm{H} 151$ is $81.05 \%$. On the other hand, H151 belonging to the cluster 7 and Hydrophobicity group used in [5] can be inferred from feature sets S1 and S2. After analyzing all candidate properties, we identify some properties in the five identified clusters for analyzing DNA-binding domains, shown in Table 11. Some typical properties discovered are hydrophobicity, secondary structure, charge, solvent accessibility, polarity, flexibility, normalized Van Der Waals volume, pK (pK-C, pK-N, pK$\mathrm{COOH}$ and $\mathrm{pK}-\mathrm{a}(\mathrm{RCOOH}))$, etc.

Most of identified properties were used in previous works $[5,6,8,9]$, but a few properties such as the flexibility property H8: BHAR880101 in cluster 7 "Average flexibility indices (Bhaskaran-Ponnuswamy, 1988)" are not utilized yet in existing method of predicting DNAbinding domains. The correlation between protein flexibility and protein function suggests a link between DNA-binding activity and the conformational freedom of the DNA-binding domain [18].

Table 8 A small, high-performance features set of size $\mathrm{c}$ from c clusters. The feature number c=5 and 8 for DNAset and DNAaset, respectively

\begin{tabular}{|c|c|c|c|c|c|c|c|c|c|c|}
\hline DNAset & ACC 83.59\% & $\begin{array}{l}\text { Cluster } \\
\text { Feature ID }\end{array}$ & $\begin{array}{l}\mathrm{C}_{7} \\
\mathrm{H} 88\end{array}$ & $\begin{array}{l}\mathrm{C}_{9} \\
\mathrm{H} 86\end{array}$ & $\begin{array}{l}\mathrm{C}_{10} \\
\mathrm{H} 67\end{array}$ & $\begin{array}{l}\mathrm{C}_{16} \\
\mathrm{H} 209\end{array}$ & $\begin{array}{l}\mathrm{C}_{18} \\
\mathrm{H} 178\end{array}$ & & & \\
\hline DNAaset & ACC $73.24 \%$ & Cluster & $C_{7}$ & $C_{9}$ & $C_{10}$ & $C_{16}$ & $\mathrm{C}_{18}$ & $C_{3}$ & $C_{14}$ & $C_{17}$ \\
\hline & & Feature ID & P159 & $\mathrm{H} 87$ & A99 & C197 & P63 & $\mathrm{H} 11$ & H396 & H451 \\
\hline
\end{tabular}

Table 9 The $m=5$ features selected from the five clusters identified from DNAset that one best feature is selected from one cluster by IGA

\begin{tabular}{cll}
\hline Feature ID & AAindex ID & \multicolumn{1}{c}{ Description } \\
\hline H88 & FAUJ880111 & Positive charge (Fauchere et al., 1988) \\
H86 & FAUJ880109 & Number of hydrogen bond donors (Fauchere et al., 1988) \\
H67 & DESM900102 & Average membrane preference: AMP07 (Degli Esposti et al., 1990) \\
C209 & NAKH920108 & AA composition of MEM of multi-spanning proteins (Nakashima-Nishikawa, 1992) \\
H178 & MEEJ800101 & Retention coefficient in HPLC, pH7.4 (Meek, 1980) \\
\hline
\end{tabular}

Table 10 The $m=8$ features selected from the eight clusters identified from DNAaset that one best feature is selected from one cluster by IGA

\begin{tabular}{cll}
$\begin{array}{c}\text { Feature } \\
\text { ID }\end{array}$ & AAindex ID & \\
\hline P159 & LEVM760107 & van der Waals parameter epsilon (Levitt, 1976) \\
H87 & FAUJ880110 & Number of full nonbonding orbitals (Fauchere et al., 1988) \\
A99 & GEIM800103 & Alpha-helix indices for beta-proteins (Geisow-Roberts, 1980) \\
C197 & NAKH900109 & AA composition of membrane proteins (Nakashima et al., 1990) \\
P63 & DAWD720101 & Size (Dawson, 1972) \\
H11 & BIOV880102 & Information value for accessibility; average fraction 23\% (Biou et al., 1988) \\
H396 & YUTK870104 & Activation Gibbs energy of unfolding, pH9.0 (Yutani et al., 1987) \\
H451 & NADH010106 & Hydropathy scale based on self-information values in the two-state model (36\% accessibility) (Naderi-Manesh et al., \\
\hline
\end{tabular}



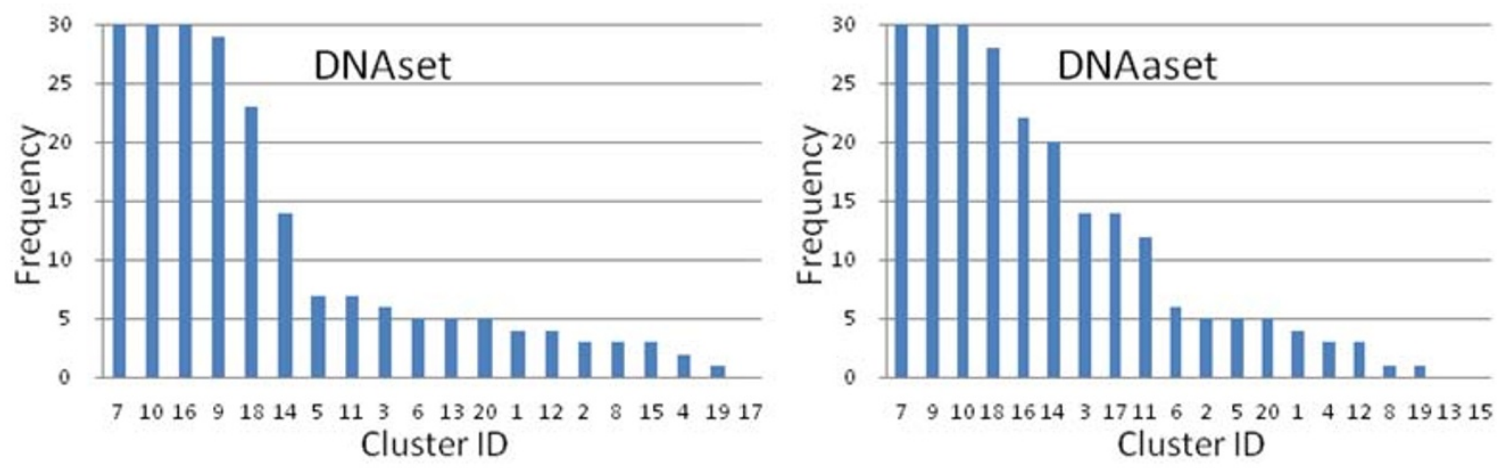

Figure 8 The appearance frequency of each identified cluster in the 30 runs. The clusters 7, 9, 10, 16 and 18 are more informative.

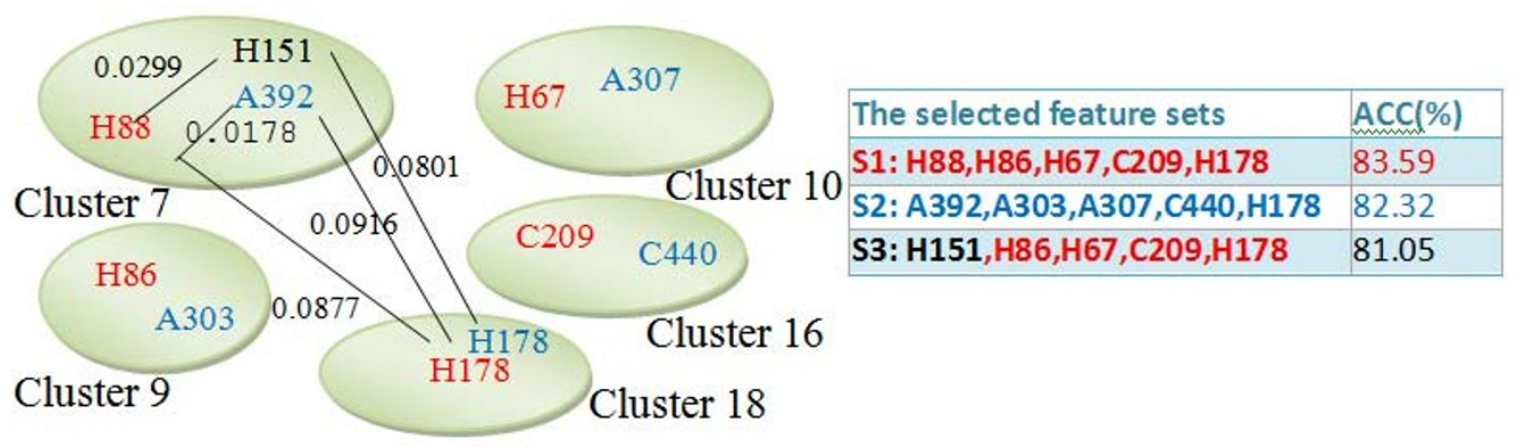

Figure 9 An illustration example for exploring promising properties. H151 can be inferred from feature sets S1 and S2.

Although most identified properties were mentioned in literature, it is not easy to know which amino acid index is the best feature in designing accurate prediction method. For example, the two feature vectors of properties H8: BHAR880101 and A:124 KARP850101 "Flexibility parameter for no rigid neighbours (Karplus-Schulz, 1985)" belonging to different clusters 7 and 9 are dissimilar. From
Table 11, it can be found that the four clusters 7, 9, 10 and 16 has properties, H115: HOPT810101 (Hydrophilicity value (Hopp-Woods, 1981)), H355: ROSM880101 (Side chain hydropathy, uncorrected for solvation (Roseman, 1988)), H398: ZIMJ680101 (Hydrophobicity (Zimmerman et al., 1968)), and H252: PRAM900101 (Hydrophobicity (Prabhakaran, 1990)), respectively, belonging to the

Table 11 Some typical properties in the five identified clusters for analyzing DNA-binding domains

\begin{tabular}{|c|c|c|c|c|}
\hline \multicolumn{2}{|c|}{$C_{\text {id }}$ AAindex ID PCP } & \multicolumn{3}{|c|}{$C_{i d}$ AAindex ID PCP } \\
\hline 7 & BHAR880101 Flexibility & 10 & FASG760105 & pK-C \\
\hline 7 & BURA740101 Secondary structure & 10 & JOND750102 & pk- $(-\mathrm{COOH})$ \\
\hline 7 & CHOC760103 Solvent accessibility & 10 & RADA880108 & Polarity \\
\hline 7 & HOPT810101 Hydrophobicity & 16 & PRAM900101 & Hydrophobicity \\
\hline 7 & FAUJ880111 Charge & 16 & FUKS010104 & Solvent accessibility \\
\hline 9 & KARP850101 Flexibility & 16 & KUMS000103 & Secondary structure \\
\hline 9 & PALJ810115 Secondary structure & 18 & PONP800107 & Solvent accessibility \\
\hline 9 & ROSM880101 Hydrophobicity & 18 & GRAR740102 & Polarity \\
\hline 9 & KUHL950101 Solvent accessibility & 18 & FASG760104 & $\mathrm{pK}-\mathrm{N}$ \\
\hline 10 & ZIMJ680101 Hydrophobicity & 18 & FAUJ880113 & $\mathrm{pK}-\mathrm{a}(\mathrm{RCOOH})$ \\
\hline 10 & EISD860101 Solvent accessibility & 18 & FAUJ880103 & Normalized van der \\
\hline 10 & GEIM800101 Secondary structure & & & Waals volume \\
\hline
\end{tabular}


Hydrophobicity group. The scenario reveals that more than one feature vectors may be needed in utilizing one physicochemical property to advance prediction accuracy.

\section{Discussion}

To avoid from overfitting the small-scale data sets in identifying physicochemical properties using an optimization approach, this study proposes a hybrid computational method of combining evidences by considering robust features and biological meanings from literature. These discovered properties in predicting and analyzing the DNA-binding mechanism can be further investigated.

In this study, the proposed approach Auto-IDPCPs aims to identify an informative feature set of physicochemical and biochemical properties, rather than proposing an accurate method for predicting DNA-binding domains/ proteins. Some techniques can be used to improve the prediction accuracy such as using ensemble classifiers with hybrid feature types and boosting techniques.

\section{Conclusions}

This study has proposed a systematic approach AutoIDPCPs to automatically identify an informative set of physicochemical and biochemical properties in the AAindex database to design SVM-based classifiers for predicting and analyzing DNA-binding domains/proteins. Since the AAindex database contains 531 physicochemical and biochemical properties of 20 naturally occurring amino acids that are the building-blocks of proteins, the proposed approach Auto-IDPCPs using an optimization method of feature selection can be useful and efficient for selecting informative physicochemical and biochemical properties, which is helpful in designing prediction methods for protein functions from sequences.

\section{Additional material}

Additional file 1: Table S1 - The 531 feature IDs used in this study and their corresponding AAindex IDs.

\begin{abstract}
Acknowledgements
The authors would like to thank the National Science Council of Taiwan for financially supporting this research under the contract numbers NSC 992221-E-009-137 and NSC 99-2627-B-009-002.

This article has been published as part of BMC Bioinformatics Volume 12 Supplement 1, 2011: Selected articles from the Ninth Asia Pacific Bioinformatics Conference (APBC 2011). The full contents of the supplement are available online at http://www.biomedcentral.com/1471-2105/12?issue=S1.
\end{abstract}

\section{Author details}

'Department of Biological Science and Technology, National Chiao Tung University, Hsinchu, Taiwan. ${ }^{2}$ Institute of Bioinformatics and Systems Biology, National Chiao Tung University, Hsinchu, Taiwan. ${ }^{3}$ Department of Multimedia Entertainment Science, Asia Pacific Institute of Creativity, Miaoli, Taiwan. ${ }^{4}$ Department of Automation Engineering, National Formosa University, Yunlin 632, Taiwan.

\section{Authors' contributions}

$\mathrm{HLH}$ designed the system, implemented programs, carried out the analysis, and participated in manuscript preparation. ICL provided biological knowledge and carried out the analysis. YFL, CTT, KTH, WLH and SJH implemented programs and participated in the experimental design. SYH supervised the whole project and participated in manuscript preparation. All authors have read and approved the final manuscript.

\section{Competing interests}

The authors declare that they have no competing interests.

Published: 15 February 2011

\section{References}

1. Gao M, Skolnick J: A threading-based method for the prediction of DNAbinding proteins with application to the human genome. PLoS Comput Biol 2009, 5(11):e1000567.

2. Shanahan HP, Garcia MA, Jones S, Thornton JM: Identifying DNA-binding proteins using structural motifs and the electrostatic potential. Nucleic Acids Res 2004, 32:4732-4741.

3. Ahmad S, Gromiha MM, Sarai A: Analysis and prediction of DNA-binding proteins and their binding residues based on composition, sequence and structural information. Bioinformatics 2004, 20:477-486.

4. Ho SY, YU FC, Chang CY, Huang HL: Design of accurate predictors for DNA-binding sites in proteins using hybrid SVM-PSSM method. Biosystems 2007, 90(1):234-241.

5. Cai YD, Lin SL: Support vector machines for predicting rRNA-, RNA-, and DNA-binding proteins from amino acid sequence. Biochim Biophys Acta 2003, 1648(1-2):127-133.

6. Fang Y, Guo Y, Feng Y, Li M: Predicting DNA-binding proteins: approached from Chou's pseudo amino acid composition and other specific sequence features. Amino Acids 2008, 34(1):103-109.

7. Kumar M, Gromiha MM, Raghava GP: Identification of DNA-binding proteins using support vector machines and evolutionary profiles. BMC Bioinformatics 2007, 8:463.

8. Shao X, Tian Y, Wu L, Wang Y, Jing L, Deng N: Predicting DNA- and RNAbinding proteins from sequences with kernel methods. J Theor Biol 2009, 258(2):289-293.

9. Yu X, Cao J, Cai Y, Shi T, Li Y: Predicting rRNA-, RNA-, and DNA-binding proteins from primary structure with support vector machines. $J$ Theor Biol 2006, 240(2):175-184.

10. Kawashima S, Pokarowski P, Pokarowska M, Kolinski A, Katayama T, Kanehisa M: AAindex: amino acid index database, progress report 2008. Nucleic Acids Res 2008, 36(Database issue):D202-205.

11. Tomii K, Kanehisa M: Analysis of amino acid indices and mutation matrices for sequence comparison and structure prediction of proteins. Protein Eng 1996, 9(1):27-36.

12. Tung CW, Ho SY: POPI: predicting immunogenicity of MHC class I binding peptides by mining informative physicochemical properties. Bioinformatics 2007, 23(8):942-949.

13. Tung CW, Ho SY: Computational identification of ubiquitylation sites from protein sequences. BMC Bioinformatics 2008, 9:310.

14. Bezdek JC: Pattern Recognition with Fuzzy Objective Function Algorithms. New York: Plenum Press; 1981.

15. Ho SY, Chen JH, Huang MH: Inheritable genetic algorithm for biobjective 0/1 combinatorial optimization problems and its applications. IEEE Trans Syst Man Cybern B Cybern 2004, 34(1):609-620.

16. Ho SY, Shu LS, Chen JH: Intelligent evolutionary algorithms for large parameter optimization problems. IEEE Trans Evolut Comput 2004, 8(6):522-541.

17. Dembele D, Kastner P: Fuzzy C-means method for clustering microarray data. Bioinformatics 2003, 19(8):973-980.

18. Gryk MR, Jardetzky O, Klig LS, Yanofsky C: Flexibility of DNA binding domain of trp repressor required for recognition of different operator sequences. Protein Sci 1996, 5(6):1195-1197.

doi:10.1186/1471-2105-12-S1-S47

Cite this article as: Huang et al:: Predicting and analyzing DNA-binding domains using a systematic approach to identifying a set of informative physicochemical and biochemical properties. BMC Bioinformatics 201112 (Suppl 1):S47. 